\title{
Plant responses to flooding
}

\author{
Chiara Pucciariello ${ }^{1}$, Laurentius A. C. J. Voesenek ${ }^{2}$, Pierdomenico Perata ${ }^{1}$ and Rashmi Sasidharan ${ }^{2 *}$ \\ 1 Institute of Life Sciences, Scuola Superiore Sant'Anna, Pisa, Italy \\ 2 Plant Ecophysiology, Institute of Environmental Biology, Utrecht University, Utrecht, Netherlands \\ *Correspondence: r.sasidharan@uu.nl
}

Edited and reviewed by:

Andreas P. M. Weber, Heinrich-Heine-Universität, Germany

Keywords: anoxia, flooding, hypoxia, low oxygen, submergence, waterlogging

Climate change models predict an increase in the frequency of flooding events globally, making flooding stress a major environmental threat for plants. Annually, crop damages due to unseasonal and severe flooding events amount to billions of dollars in yield losses. Despite the vulnerability of most crops to wet conditions, there is significant variation in the plant tolerance to flooding. Plant species adapted to wet areas have evolved specific strategies to deal with and even thrive under these conditions. In order to generate flood resilient, high yielding crops, it is of essence to not only understand the different elements that define flooding stress, but also to determine how plants sense and respond to these signals. In addition, the understanding of the genetic basis of tolerance variation and the underlying genes and processes will be key to discovering novel tolerance mechanisms and ultimately translating these to crops. In the last few years, the flooding research community has made leaps and bounds in understanding several of these aspects, unraveling more layers of the plant flooding response and paving the way for further research. This special issue entitled "Plant responses to flooding stress" brings together a collection of review and original research articles reflecting the broad scope and dynamics of the field of plant anaerobiosis.

The conditions during flooding can drastically affect survival. Large survival differences exist between plants submerged in complete darkness versus those submerged with some light, hinting at the importance of underwater photosynthesis. This importance is not only related to the production of carbohydrates but also, to the generation of molecular oxygen that accumulates in submerged plants and diffuses to tissues with less oxygen (e.g., roots). Pedersen et al. (2013) summarize recent advances and methods to quantify underwater photosynthesis in terrestrial plants in relation to leaf acclimations.

Among cereals, rice has the unique capacity to germinate and grow vigorously in flood-prone areas. Rice adaptive plasticity to different hydrological regimes has allowed the selection and characterization of some genotypes that have been subsequently used to breed high yielding and tolerant modern varieties. Miro and Ismail (2013) present an overview of the current understanding of the mechanisms associated with tolerant traits such as anaerobic germination and early vigor in rice that can help to develop tolerant varieties for direct-seeding systems. Narsai and Whelan (2013) employed a meta-analyses of microarray data to compare global low oxygen transcriptomic responses of tolerant rice with the relatively intolerant Arabidopsis. Based on their results they conclude that while Arabidopsis is simply responding to general stress conditions, rice displays transcriptome reconfigurations specific to low oxygen conditions.

Energy limitation during hypoxia, necessitates several resource limiting measures in plants. Amongst these is the use of pyrophosphate (PPi)-dependent enzymes that can take PPi as a substrate to catalyze reactions, thus sparing precious ATP reserves. Mustroph et al. (2013), execute a detailed expression analyses on the rice ATP and PPi-dependent phosphofructokinase gene family to establish their role during anoxic stress.

The study of flood adaptive strategies that evolved in nature and were selected by farmers will be crucial to develop stresstolerant crop varieties. In this context, other environmental factors likely influence submergence tolerance, and stress combinations need to be explored toward the selection of crops tolerant to multiple stresses. Zeng et al. (2013) studied the combined response to waterlogging and salinity of barley varieties. They conclude that hypoxia is not the only factor determining the differential response of the genotypes to the stress combination and that the soil type strongly influence the presence of elemental toxicity.

The accumulation of toxic substances in flooded soils is a serious concern for plants. In some plant species, the formation of an apoplastic suberin barrier in the roots can help to prevent toxins entry and at the same time loss of oxygen. Watanabe et al. (2013) review the role of this barrier in waterlogging tolerance. In a similar context, Lamers et al. (2013) provide an overview of the effect of toxic sulfide accumulation in fresh and marine water. The accumulation of sulfide by microbial activity occurring in aquatic ecosystems is exacerbated by anthropogenic inputs. This makes sulfide-related pollution an urgent question to address.

Reactive oxygen species (ROS) are a major component of low oxygen stress. ROS production is expected to contribute to submergence adaptation. Owing to their transient nature ROS detection has been famously difficult to accurately quantitate. Steffens et al. (2013) outline the role of ROS during flooding and review the potential of techniques such as electron paramagnetic resonance to investigate ROS in planta.

Large trees encounter specific problems such as relatively deep floods (10-15 m) and prolonged durations of these floods (up to 7 months). Herrera (2013) reviews the biochemical responses of tropical trees to flooding in South America. The emphasis in this overview lies on leaf gas exchange, stomatal conductance, water status, and carbohydrate balance.

Beside plants, the unicellular green alga Chlamydomonas reinhardtii has received special attention for its response to low 
oxygen due to its capacity to produce $\mathrm{H}_{2}$, a source of renewable energy, under anoxia. Catalanotti et al. (2013) describe the fermentation pathway of Chlamydomonas, also discussing the fermentation process evolution.

This Frontiers special showcases the broad scope and importance of low oxygen stress studies in plants. We are grateful to all the authors for contributing to this collection. We would also like to acknowledge all the reviewers for taking the time to assess the work submitted here and helping to shape this focus issue.

\section{REFERENCES}

Catalanotti, C., Yang, W., Posewitz, M. C., and Grossman, A. R. (2013) Fermentation metabolism and its evolution in algae. Front. Plant Sci. 4:150. doi: 10.3389/fpls.2013.00150

Herrera, A. (2013) Responses to flooding of plant water relations and leaf gas exchange in tropical tolerant trees of a black-water wetland. Front. Plant Sci. 4:150. doi: $10.3389 /$ fpls.2013.00106

Lamers, L. P. M., Govers, L. L., Janssen, I. C. J. M., Geurts, J. J. M., Van der Welle, M. E. W., Van Katwijk, M. M., et al. (2013). Sulfide as a soil phytotoxin - a review. Front. Plant Sci. 4:268. doi: 10.3389/fpls2013.00268

Miro, B., and Ismail, A. M. (2013). Tolerance of anaerobic conditions caused by flooding during germination and early growth in rice (Oryza sativa L.). Front. Plant Sci. 4:269. doi: 10.3389/fpls. 2013.00269

Mustroph, A., Stock, J., Hess, N., Aldous, S., Dreilich, A., and Grimm, B. (2013) Characterization of the phosphofructokinase gene family in rice and its expression under oxygen deficiency stress. Front. Plant Sci. 4:125. doi: 10.3389/fpls.2013.00125

Narsai, R., and Whelan, J. (2013). How unique is the low oxygen response? An analysis of the anaerobic response during germination and comparison with abiotic stress in rice and Arabidopsis. Front. Plant Sci. 4:349. doi: 10.3389/fpls.2013.00349

Pedersen, O., Colmer, T. D., and Sand-Jensen, K. (2013) Underwater photosynthesis of submerged plants recent advances and methods. Front. Plant Sci. 4:140. doi: 10.3389/fpls.2013.00140

Steffens, B., Steffen-Heins, A., and Sauter, M. (2013) Reactive oxygen species mediate growth and death in submerged plants. Front. Plant Sci. 4:179. doi: 10.3389/fpls.2013.00179

Watanabe, K., Nishiuchi, S., Kulichikhin, K., and Nakazono, M. (2013) Does suberin accumulation in plant roots contribute to waterlogging tolerance? Front. Plant Sci. 4:178. doi: 10.3389/fpls. 2013.00178

Zeng, F., Shabala, L., Zhou, M., Zhang, G., and Shabala, S. (2013). Barley responses to combined waterlogging and salinity stress: separating effects of oxygen deprivation and elemental toxicity. Front. Plant Sci. 4:313. doi: $10.3389 /$ fpls. 2013.00313

Conflict of Interest Statement: The authors declare that the research was conducted in the absence of any commercial or financial relationships that could be construed as a potential conflict of interest.

Received: 02 May 2014; accepted: 05 May 2014; published online: 23 May 2014.

Citation: Pucciariello C, Voesenek LACJ, Perata P and Sasidharan R (2014) Plant responses to flooding. Front. Plant Sci. 5:226. doi: 10.3389/fpls.2014.00226

This article was submitted to Plant Physiology, a section of the journal Frontiers in Plant Science.

Copyright (c) 2014 Pucciariello, Voesenek, Perata and Sasidharan. This is an openaccess article distributed under the terms of the Creative Commons Attribution License (CC BY). The use, distribution or reproduction in other forums is permitted, provided the original author(s) or licensor are credited and that the original publication in this journal is cited, in accordance with accepted academic practice. No use, distribution or reproduction is permitted which does not comply with these terms. 